\title{
CONSTITUCIÓN, FEMINISMO Y DIVERSIDAD. EL TRILEMA DEL FEMINISMO CONSTITUCIONAL
}

\author{
CONSTITUTION, FEMINISM AND DIVERSITY.
}

PABLO DE LORA DELTORO*

\section{Resumen}

Este trabajo tiene por objeto analizar qué quiere decir que una Constitución - o para el caso, una política pública o una pieza legislativaadopte una "perspectiva de género"; qué pueda querer decir que una Constitución es "feminista" y si tal cosa es deseable, y qué razones pueden justificar que la composición de la Convención Constitucional sea paritaria entre hombres y mujeres.

\section{Palabras Clave}

Constitución, feminismo, feminismo constitucional.

\begin{abstract}
The objective of this paper is to analyze possible meanings when a Constitution - or for that matter a public policy or a piece of legislation - adopts a "gender perspective"; what could it mean that a Constitution is "feminist" and if such a thing is desirable, and what reasons can justify the composition of the Constitutional Convention being equal between men and women.
\end{abstract}

Artículo recibido para su evaluación el 30 de octubre de 2020, y aprobado para su publicación el 20 de diciembre de 2020.

" Doctor en derecho, Universidad Autónoma de Madrid. Profesor de Filosofía del Derecho. Universidad Autónoma de Madrid. Email: pablo.delora@uam.es. 


\section{Key Words}

Constitution, feminism, feminism constitutional.

\section{INTRODUCCIÓN.}

El pasado 26 de octubre de 2020, y por una amplia mayoría, la ciudadanía chilena ha decidido iniciar un proceso constituyente que contará con una Convención Constitucional que necesariamente habrá de ser paritaria entre hombres y mujeres, de acuerdo con lo establecido por el Congreso y el Senado chilenos a principios del mes de marzo de $2020^{1}$. Será la primera convención constitucional en la historia que cuente con tal representación.

Muchos han sido los que han visto en este hito un primer paso para la necesaria consecución de un texto constitucional "feminista", "con perspectiva de género", un documento que habrá de representar mejor los intereses de las mujeres, y no solo los de las mujeres. Rosa Moreno, presidenta del Partido Alternativa Feminista, el primer partido feminista de la historia moderna de Chile, decía a este respecto: "Es la primera vez que se escribirá una Constitución en Chile a partir de una hoja en blanco y creemos que el feminismo puede definir un nuevo tipo de sociedad, organizada desde la base social, desde los territorios, con participación real y paritaria, fundada en principios como la inclusión, la no-violencia, la no discriminación y el respeto por el medio ambiente"2. Por su parte, Catalina Lagos Tschorne ha afirmado: "Incluir el enfoque de género -de comienzo a fin- en la nueva Constitución es importante y necesario, pues permitirá corregir las invisibilizaciones y exclusiones del pasado y porque tiene el potencial de producir cambios reales en la vida de las mujeres y de las identidades o sexualidades disidentes y acelerar la consecución de la igualdad real y efectiva para ellas y elles"3.

En este breve ensayo me propongo elucidar: a) qué pueda querer decir que una Constitución - o para el caso una política pública o una pieza legislativa- adopte una "perspectiva de género"; b) qué pueda querer decir que una Constitución es "feminista" y si tal cosa es deseable, y c) qué razones

1 Se trata de la Ley $N^{\circ} 21.216$ promulgada el 20 de marzo de 2020.

2 https://www.pressenza.com/es/2020/03/entrevista-a-rosa-moreno-presidenta-delpartido-alternativa-feminista-queremos-una-nueva-constitucion-en-la-que-se-expresenuestra-mirada/ (última consulta el 9 de noviembre 2020). 
pueden justificar que la composición de la Convención Constitucional sea paritaria entre hombres y mujeres. Las distintas secciones en las que se divide este ensayo abordarán esas preguntas en el orden enunciado y al final esgrimiré una conclusión en la forma de un trilema.

\section{II. ¿UNA CONSTITUCIÓN CON PERSPECTIVA DE GÉNERO?}

Desde hace años se escucha en el debate académico y en la esfera pública la expresión "perspectiva de género" como un necesario enfoque que habría de informar el estudio y análisis del Derecho, el de otros muchos fenómenos sociales, y, para lo que aquí interesa más, la política pública y legislativa. Sin embargo, la expresión, a poco que uno rastree sus usos, es equívoca y puede querer decir, al menos, lo siguiente.

2.1. El recordatorio o vindicación del protagonismo de las mujeres en algún ámbito relevante y de cómo la historia las ha opacado. En este primer sentido es frecuente apelar al papel que desempeñaron algunas mujeres en la consecución de avances y logros importantes en dominios varios del saber humano (la ciencia, las artes, el conocimiento en general), o de cómo su subordinación, si es que no explotación, fue coadyuvante a que se alcanzaran esos hitos. Tomen por ejemplo el papel decisivo que jugó la química Rosalind Franklin para que pudiera describirse la doble hélice de la molécula del ADN, o cómo J. Marion Sims, considerado por muchos como "el padre de la ginecología moderna", pudo desarrollar las técnicas quirúrgicas que permiten operar la fístula vesico-vaginal (una condición terriblemente molesta y degradante para la mujer que la sufre por la incontinencia urinaria que causa) "gracias" al empleo de esclavas negras como "sujetos de experimentación".

2.2. Por perspectiva de género puede en segundo lugar quererse aludir a la especificación o concreción de ciertos principios o nociones compartidas por "razón de género". Tomemos por ejemplo la agravante de alevosía en los delitos de violencia doméstica, o las condiciones exigibles para la justificación basada en la legítima defensa en el mismo ámbito de la agresión de la pareja. Tanto en un caso como en otro podemos incorporar modulaciones que atienden al género, hasta el punto, por ejemplo, de admitir una suerte de ataque preventivo de la mujer como forma de legítima 
defensa frente al maltratador varón habitual, o formas de comisión alevosa del delito que sólo se habrán de aplicar a los hombres ${ }^{4}$.

2.3. En tercer lugar, un enfoque de género puede suponer el análisis y estudio del modo en el que la práctica regulatoria o una política pública afecta de una manera particular a grupos o colectivos determinados, para el caso las mujeres, y, finalmente;

2.4. La perspectiva de género puede implicar una apuesta por la implementación de políticas de "diferencia", es decir, distinciones de trato, prerrogativas o discriminaciones "positivas" en favor de las mujeres que suponen el sacrificio de valores o principios importantes. La mayor punición en España de ciertos delitos vinculados a la violencia de género cuando el infractor es un hombre y la víctima su pareja o expareja es un supuesto suficientemente claro.

Ninguno de estos usos de la expresión que acabo de destacar atribuye a la mirada o perspectiva de género ninguna suerte de "privilegio epistémico" sobre la realidad, incluyendo el análisis, conformación o transformación de la realidad jurídica, si bien soy consciente de que no faltan en la literatura quienes defienden que las mujeres sí tienen una peculiar, única e intransferible forma de estar en el mundo ${ }^{5}$. No discutiré aquí esa manifestación del "feminismo de la diferencia", las credenciales científicas de semejante propuesta teórica, pues excede de mis propósitos en este artículo ${ }^{6}$. Me basta sencillamente con indicar el aire de paradoja que tiene esta tesis de la "razón situada" - no solo por circunstancias tales como el género sino también por la identidad racial o étnica: ¿cómo entender esos accesos únicos o privilegiados al conocimiento, esas experiencias o vivencias intransferibles, con el hecho fácilmente constatable de que esa "metateoría" o propuesta epistemológica es formulada también por hombres? ¿Por qué pueden los hombres abordar "cosas de mujeres", o las mujeres implicarse en "problemas de hombres"? ¿Cómo habríamos de entender que el liberal inglés John Stuart Mill escribiera en 1869 en favor

4 Véase, a este respecto, el estudio de CORREA FLOREZ, María: Legítima defensa en situaciones sin confrontación: la muerte del tirano de casa. Colección Ciencias Penales, Grupo Editorial Ibáñez, Universidad de los Andes, Bogotá, 2017.

5 Véase el clásico de los estudios de género de FIELD BELENKY, Mary, MCVICKER CLINCHY, Blythe, RULE GOLDBERGER, Nancy, y MATTUCK TARULE, Jill: Women's Ways of Knowing: The Development of Self, Voice, and Mind, Basic Books, New York, 1986.

6 Puede verse, por todos, PINKER, Steven: The Better Angels of Our Nature. Penguin, Nueva York, 2011. pp. 342 ss. 
del sufragio femenino y la socialista española Victoria Kent se opusiera en el Parlamento de la República en 1931?

\section{III. ¿UNA CONSTITUCIÓN FEMINISTA?}

Me voy a detener en particular en el último de los sentidos que he presentado del sintagma "perspectiva de género" - aquel que alude a la diferenciación sustancial o al sacrificio de algún principio o valor importante en favor de las mujeres- si bien antes sí quería apuntar que, en relación a la "especificación" de principios en función del género, he de recordar que las razones de tal concreción pueden ser siempre descritas con tal nivel de generalidad que no sean "exclusivas del género". La modulación de la legítima defensa o la alevosía tienen que ver con la posición de mayor vulnerabilidad en la relación de pareja, lo cual también puede concurrir en los hombres - aunque sea estadísticamente menos frecuente. En definitiva, la perspectiva de género, cuando se usa para la especificación de un principio, regla, criterio o concepto, o bien para tomar en consideración ciertas realidades "de género", exige la determinación de un colectivo - el de las mujeres- mediante condiciones individualmente necesarias y conjuntamente suficientes, cosa que resulta imposible. Cuando se predica, por ejemplo, que adoptando una perspectiva de género se bloquea la posibilidad de toda política tendente a limitar o eliminar las pensiones no contributivas de viudedad, se olvida que hay viudas multimillonarias y también pensionistas varones que reciben pensiones contributivas y aun así viven en el umbral de la pobreza. De otra parte, y en relación al tercero de los sentidos de la noción "perspectiva de género", conviene llamar la atención de que cuando se tienen en cuenta divisiones de roles o estereotipos para proceder al diseño de políticas públicas o regulaciones sectoriales "por razones de género", hay que ser conscientes de que con ello se puede estar contribuyendo a reforzar dichos patrones prejuiciosos. Así, sostener que la planificación urbanística debe tener en cuenta la perspectiva de género y dotar las calles de aceras más anchas porque las mujeres son las que llevan los carros de bebé, refuerza un estereotipo: los hombres no nos ocupamos de esos quehaceres.

Si nos preguntamos qué caracteriza a una "Constitución feminista" tal vez lo primero que debamos plantearnos es qué caracteriza, en primer lugar, a esa norma jurídica a la que nos referimos como "Constitución", y, 
a continuación, qué identifica al feminismo ${ }^{7}$. Y no, no podré acometer en estas breves reflexiones semejante empresa. Me bastará con tomar el tweet lanzado por la organización "ONU Mujeres" el 28 de enero de $2020^{8}$. Allí se preguntaba: “ ¿Cree usted que hombres y mujeres deben tener iguales derechos y oportunidades? Si responde que sí, enhorabuena es usted un feminista. Si la respuesta es no, siga intentándolo". El feminismo que todos deberíamos abrazar, de acuerdo con la ONU, es una especificación del principio de la igual consideración de intereses: todos debemos contar como uno y nadie más que como uno, tal y como instruía el padre del utilitarismo contemporáneo, Jeremy Bentham, o, más recientemente, Ronald Dworkin al apostar por la fórmula del "equal concern and respect". Por supuesto, que las mujeres cuenten también como "uno", no como menos que uno, o como nada, es una excepcionalidad histórica. Incontables códigos, leyes, reglas de trato social y mecanismos informales han propiciado que las mujeres vivan de manera subordinada, pero, ¿podría ser eso hoy posible en Chile? ¿Podría, en ese sentido ser la futura Constitución chilena una Constitución "no feminista"? Absolutamente no. Como al respecto de la propia historia chilena, sus compromisos con los tratados internacionales y cánones jurídicos bien arraigados, no hay una posible "hoja en blanco" en relación con las mujeres y su condición ciudadana. Pero tampoco la debería haber en un sentido diametralmente opuesto: por ejemplo, la plasmación constitucional de estándares de prueba mediante el privilegio del "testimonio de las mujeres" en aquellos supuestos - señaladamente los vinculados a los delitos contra la libertad sexual, las relaciones familiares o la violencia de género- en los que el hombre es el acusado ${ }^{9}$. Tal cosa

$7 \quad$ Hay un obvio sentido en el que una Constitución responde a una demanda feminista y es la de usar un lenguaje "inclusivo". Para un análisis de dicha exigencia me remito a los extraordinarios estudios que sobre la materia ha hecho Alfonso García Figueroa dónde muestra cuán desorientadas desde el punto de vista lingüístico pueden resultar tales demandas, así como en general la insistencia en el uso del lenguaje inclusivo: "Constitución feminismo de Estado y convenciones lingüísticas (I, II y III)", publicado en Almacén de Derecho los días 26 de enero, 28 de enero y 4 de febrero (https://almacendederecho. org/constitucion-feminismo-de-estado-y-convenciones-linguisticas-i, https:// almacendederecho.org/constitucion-feminismo-de-estado-y-convenciones-linguisticasii y https://almacendederecho.org/constitucion-feminismo-de-estado-y-convencioneslinguisticas-y-iii). Véase igualmente GARCÍA FIGUEROA, Alfonso: "Feminismo de Estado: fundamentalmente religioso y religiosamente fundamentalista". En: Eunomía. Revista en Cultura de la Legalidad, 17, 2019. Especialmente pp. 364-367.

8 https://twitter.com/UN_Women/status/1222075599713374208

9 Véase, por todas, GIMENO, Beatriz: "Cambiarlo todo". En: Ctxt, №158, 2018, febrero (https://ctxt.es/es/20180228/Firmas/18208/Feminismo-izquierda-puritanismoneoliberalismo-Beatriz-Gimeno.htm). 
supondría liquidar un supuesto constitucional civilizatorio de calibre parecido al principio de igualdad: la presunción de inocencia que exige condenar penalmente cuando se haya producido prueba de cargo suficiente como para disipar toda duda razonable (tal y como se expresa en el ámbito anglosajón: beyond any reasonable doubt).

¿Debería la Constitución chilena, o cualquier otra, incluir un derecho a vivir una vida "libre de violencias" como una exigencia feminista ${ }^{10}$ ? ¿No es ya lo que ocurre en cualquier Constitución cuando consagra derechos humanos tan básicos como el derecho a la vida o a la integridad corporal?

En las respuestas dadas al mencionado tweet de ONU Mujeres destaca un mensaje en el que una tuitera señala que el feminismo es la lucha de las mujeres para liberarse de la opresión de los hombres, y que lo que ONU Mujeres describe es "igualitarismo", una lucha que merece la pena pero que no es feminismo.

La opresión ejercida contra las mujeres, viene a señalarse por el feminismo preponderante en estos tiempos, el feminismo que destila la respuesta de nuestra tuitera, es el resultado de una cierta estructura social: el patriarcado (o heteropatriarcado). Se trata de una estructura que se apuntala sobre determinadas "brechas", es decir, la constatación estadística de que en algún ámbito - ocupación de posiciones sociales relevantes, disfrute de bienes primarios o satisfacción de intereses o necesidades- las mujeres, aunque disfrutan de la plena igualdad formal, están peor que los hombres.

Esta invocación de la estructura patriarcal, empero, tiene que sortear tres obstáculos, o, si se quiere, satisfacer tres requisitos para ser persuasiva:

a) ¿Cómo computar, componer, operacionalizar las muy distintas brechas que pueden ser predicadas entre individuos diversos en algún aspecto, o discriminar entre esas diferencias? Pensemos por ejemplo en la comparación de indicadores socioeconómicos entre las mujeres chilenas en núcleos urbanos y los hombres que pertenecen al pueblo originario Mapuche. Son porcentualmente más las mujeres muertas a manos de su pareja que los hombres muertos a manos de su pareja mujer, o violadas. La evidencia resulta abrumadora al respecto, pero son más, muchos más, los hombres que mueren en accidente laboral, o los que cumplen penas privativas de libertad o las víctimas -sin más- de la violencia física; es

10 Así lo sostiene, GÓMEZ FERNÁNDEZ, Itziar: “¿Qué es eso de reformar la Constitución con perspectiva de género? Mitos caídos y mitos emergentes a partir del libro Una constituyente feminista”. En: Eunomía. Revista en la Cultura de la Legalidad, Vol. 16, 2019. p. 320 . 
menor -y además de manera universal- su esperanza de vida, y mayor su fracaso escolar en los estudios de secundaria.

b) Centrémonos en la que puede tildarse como la más célebre de todas las brechas: la brecha salarial. La discriminación salarial por razón de sexo está prohibida en la inmensa mayoría de las legislaciones del mundo, también en Chile. De nuevo, pagar distinto salario por el mismo trabajo por el hecho de ser mujer quiebra un ideal de igualdad universalmente asumible. Y sin embargo, los hombres, en promedio, ganan más que las mujeres, cotizan más y por tanto disfrutan de mejores pensiones. So far so good, pero, ¿por qué esa desigualdad constituye una injusticia ${ }^{11}$ ?

De otra parte: ¿por qué el salario sería el "resultado" a medir que evidencia la desigualdad y no otras métricas? De nuevo, si tenemos en cuenta esas otras métricas la consecuencia pudiera ser tal vez que la brecha opera en el sentido inverso al pensado: en perjuicio de los hombres. La brecha, por ejemplo, puede estar causada por la ocupación en empleos más penosos y peligrosos que por ello se pagan más, pero en los que se sufre más. Al fin: ¿qué principio justifica que la clase hombres y la clase mujeres ganen lo mismo? No solemos decir que todos los individuos deben ganar lo mismo por razones de justicia. Si hay brechas salariales entre grupos étnicamente diferenciados, ¿deben cerrarse por necesariamente injustas?

c) Pero tal vez todo lo anterior es el resultado de una cierta miopía analítica. Descartado que podamos apelar a una desigual distribución "natural" del talento entre hombres y mujeres, y estando todas las ocupaciones y posiciones abiertas a todos, resulta, como nos señalan los estudiosos, que las mujeres son más proclives a sacrificar su carrera profesional y piden más excedencias o tiempos parciales por razones familiares. Claro que si todas esas son finalmente "elecciones" el resultado último no es de manera inmediatamente obvia la muestra de opresión o injusticia alguna. ¿O no son elecciones, planes de vida autónomamente asumidos los de tener familia en primer lugar y dedicar más tiempo a ella que al desarrollo profesional? Si no lo son urge preguntarnos si no tendremos entonces que abolir la institución familiar tal y como la conocemos, según proponía célebremente Simone de Beauvoir y no pocas feministas que ven en la familia uno de los más obvios elementos de la estructura patriarcal que subyuga a las mujeres.

11 Así, RADCLIFFE-RICHARDS, Janet: “Only X\%: The Problem of Sexual Equality”. En: Journal of Practical Ethics, Vol. 2, Issue 1, 2014. 
Pero tal vez no haya que llegar a tal extremo, sino más bien intervenir en la vida íntima, privada y familiar de los individuos, al modo en el que muchas teóricas políticas del feminismo - señaladamente Susan Moller Okin (1989) - han proclamado bajo la consideración de que "lo personal es político". Se trataría de regular la conciliación y reparto de las tareas domésticas, obligar al disfrute paritario de los permisos de paternidad y maternidad, y otras medidas similares que coadyuvarían a que las mujeres pudieran compatibilizar mejor su trabajo con su aspiración de ser madres. Parafraseando el lema clásico del feminismo que acabo de recordar, se trataría de invocar que "lo personal es constitucional".

La cuestión obvia que sale al paso es cuán personal ha de serlo. Muchas feministas consideran, a ese respecto, que una Constitución feminista debe consagrar los derechos sexuales y reproductivos, lo cual incluye la posibilidad del aborto más o menos irrestricto ${ }^{12}$, pero al tiempo otras sostienen que la maternidad ha de consagrarse constitucionalmente como de interés público ${ }^{13}$. ¿Qué se quiere decir con ello? Constitucionalizar el fomento de la maternidad a partir de la consideración de que la reproducción es socialmente deseable, no es, por supuesto, solo un asunto de mujeres - de momento, y a la espera de avances en materia de reproducción asistida que permitan la procreación sin pareja- sino que, además, no necesariamente genera externalidades sociales positivas, con lo que no está justificado en todo caso que se dediquen recursos públicos a lo que entonces constituirá una pura preferencia - la de tener hijos- de satisfacción privada.

Por otro lado, las cuestiones que se vinculan al derecho a la maternidad no son, obvio es decirlo, socialmente pacíficas, ni siquiera entre las propias feministas y ni siquiera al respecto de la interrupción voluntaria del embarazo y sus posibles condicionantes. Resulta pues que lo que sea esencialmente feminista es esencialmente contestable, por decirlo con la

12 Así, RODRÍGUEZ PALOP, María Eugenia: "Esta Constitución no es nuestra. Propuestas para una Constitución feminista”. En: Escudero Alday, Rafael y Martín, Sebastián (eds.): Fraude o esperanza: 40 años de la Constitución. Akal, Madrid, 2018. pp. 140-154. En el ámbito de la discusión constituyente chilena, la abogada Lorena Fries ha afirmado que: “... nosotras seguimos afirmando que tenemos soberanía sobre nuestros cuerpos y, por lo tanto, tenemos la autonomía para decidir cuándo, cómo, con quién, de qué manera queremos tener o no hijos y esa libertad de decidir tiene que estar consagrada"; véase "La lucha feminista por incluir los nuevos derechos sexuales y reproductivos en la nueva Constitución", 2 de septiembre de 2020 (https://radio.uchile.cl/2020/09/02/la-luchafeminista-por-incluir-los-derechos-sexuales-y-reproductivos-en-la-nueva-constitucion/, última visita el 9 de noviembre de 2020).

13 GÓMEZ FERNÁNDEZ, cit., p. 320. 
célebre fórmula de W. B. Gallie (1955) al respecto de ciertos conceptos políticos. Consagrar los derechos reproductivos, ¿debería incluir también la "constitucionalización" de la gestación de sustitución, ya sea para prohibirla, opción por la cual abogan muchas feministas ${ }^{14}$, o para permitirla expresamente como una manifestación del muy feminista principio "mi cuerpo mis reglas", el mismo principio, por cierto, que permite amparar la licitud jurídica del aborto como señala Lorena Fries ${ }^{15}$ ?

Como reivindicaciones para una agenda constituyente feminista se han propuesto también el laicismo, la garantía de los suministros de los consumos vitales básicos que deberían considerarse como bienes comunes, o la igual protección efectiva de derechos civiles y políticos y sociales y económicos ${ }^{16}$. Todos ellos son ideales emancipatorios de la condición humana en general, o ideales políticos genéricos, o como, siguiendo al filósofo John Rawls, formas de especificar la justicia como primera virtud de las instituciones sociales, que ni son específicamente feministas ni han de engrosar necesariamente el contenido de una Constitución útil y duradera para una sociedad plural como la chilena, un país en el que, como en tantos otros, reina un desacuerdo moral razonable sobre muchos asuntos públicos que habrán de ser resueltos en el juego de la política ordinaria, aun cuando se acepte que algunos derechos y la organización del poder mismo operen como "cartas de triunfo" por usar la manida expresión de Ronald Dworkin.

$\mathrm{Y}$ es en este punto donde se impone hacer alguna consideración al respecto de algo que dejé anteriormente apuntado: la idea de Constitución misma, sin apellidos, que traslucen las propuestas de reforma o inclusión constitucional desde ciertos sectores del feminismo. Tomemos la garantía de los suministros de consumos vitales básicos a los que antes aludía, u otras especificaciones que se han ido abriendo paso en la legislación ordinaria - en materia de violencia de género $^{17}$, por ejemplo, o la prohibición de la educación segregada en la escuela ${ }^{18}$ - y que ahora se aspira a constitucionalizar. Siguiendo una distinción consolidada en la doctrina constitucional, se trata con ello de promulgar una Constitución de "detalle" que corre el peligro de ser percibida en el futuro más como lastre que como

14 Incluyendo a RODRÍGUEZ PALOP, María Eugenia: “Argumentos contra la gestación por sustitución”. En: García Manrique, Ricardo, (ed.): El cuerpo diseminado. Estatuto, uso y disposición de los biomateriales humanos. Aranzadi, Navarra, véase 2018a.

15 Vid., supra nota 12.

16 RODRÍGUEZ PALOP, "Esta Constitución no es nuestra...”, cit., pp. 140-154.

17 Ibídem, pp. 148-149.

18 GÓMEZ FERNÁNDEZ, cit., pp. 320 y ss. 
un dique "contramayoritario", y que, además, puede partir de entrada con un insuficiente consenso social.

\section{IV. ¿UNA CONSTITUCIÓN FEMINISTA COMO CONSTITUCIÓN PARITARIA?}

El sentido más diáfano en el que un constituyente actuaría impelido por un enfoque de género con el afán de plasmar una concepción feminista del poder político es el de constitucionalizar alguna "política de la diferencia", un privilegio, prerrogativa o discriminación positiva en favor de las mujeres, bien por la necesidad de revertir las discriminaciones habidas en el pasado, bien por razones vinculadas a la "representatividad-espejo", en la terminología de Hanna Pitkin. La más clara medida es tanto la participación paritaria en el proceso constituyente, como ocurrirá en Chile, cuanto la obligación de implantación de paridades futuras en las instituciones públicas o incluso en el ámbito privado (consejos de administración en sociedades mercantiles, etc. ${ }^{19}$.

En cuanto a lo primero, es frecuente advertir que las Constituciones en cuya elaboración han participado fundamentalmente hombres no son representativas de las mujeres. Hay una suerte de "pecado original" 20 , pecado que ahora se estaría expiando en el proceso constituyente chileno ${ }^{21}$.

Vale la pena, por comparación, recordar, siquiera sea sumariamente, lo que pasó en el proceso constituyente español, y ello por razones obvias de vinculación cultural y de cercanía histórica.

En el debate español de 1978 no faltaron voces en el seno del feminismo que denunciaron el proyecto de Constitución por escasamente "feminista". Así, en su edición de 29 de noviembre de 1978, a una semana de la celebración del referéndum de aprobación de la Constitución española, el ya entonces muy influyente periódico El País informaba de que la Plataforma

19 Ibídem, pp. 325-329 y RODRÍGUEZ PALOP, “Esta Constitución no es nuestra ...”, cit., p. 143.

20 Véase GÓMEZ FERNÁNDEZ, cit., p. 317 y ss. y RODRÍGUEZ PALOP, "Esta Constitución no es nuestra ...”, cit., pp.137 ss.

21 Para una historia de la progresiva incorporación global de las mujeres a los procesos constituyentes, resulta fundamental el libro de RUBIO-MARÍN, Ruth y IRVING, Helen (2019): "Women as Constitution-Makers: The Promises and the Challenges of Participation". En: Rubio-Marín, Ruth y Irving, Helen (edits): Women as ConstitutionMakers. Case Studies From the New Democratic Era. Cambridge University Press, Cambridge-Nueva York, 2019. 
de Organizaciones Feministas de Madrid había emitido un comunicado en el que se criticaba agriamente el texto constitucional, “... peor [para las mujeres actuales] que para las abuelas de 1931"22. Los ecos de esas voces se escuchan hoy en las gargantas de algunas teóricas del feminismo que entonces contaban pocos años, si es que habían llegado a nacer.

Para calibrar lo sorprendente que puede resultar la afirmación de antaño y sus reverberaciones de hogaño, basta con consignar que días después, el 3 de diciembre de ese mismo año de 1978, se podía leer una manifiesto - "Mujeres ante la Constitución"- en el mismo periódico, en el que se defendía, en cambio, el voto afirmativo a la Constitución puesto que: “... en el momento actual, por encima de intereses de grupo, aunque éste englobe a la mitad de la población, es urgente garantizar un marco de convivencia para todos los españoles. Este marco es la Constitución". Y es que, se añadía: “... esta Constitución tiene artículos que podrían ser mejorados, no sólo los citados por las declaraciones aludidas, sino otros que afectan a la totalidad de los ciudadanos, pero, como todo el mundo sabe, es el resultado de mutuas concesiones inspiradas en el deseo de convivencia. En consecuencia, ningún grupo tiene su Constitución, pero ésta puede ser la de todos los españoles"23.

El manifiesto, interesa mucho recordarlo, incluye la firma de significadas mujeres de la izquierda o la extrema izquierda como Dolores Ibarruri, Teresa Pamiès, Pilar Bravo o Lidia Falcón, pero también de otras tradiciones políticas - Soledad Becerril, Consuelo de la Gándara, Blanca de la Cierva por nombrar unas cuantas - cubriendo un amplio espectro ideológico. Para cualquiera mínimamente conocedor de la historia contemporánea española ha de resultar elocuente encontrar que nada más y nada menos que a "La Pasionaria" solicitando que las mujeres apoyaran la que finalmente es la Constitución española actualmente vigente ${ }^{24}$.

22 La referencia es obviamente a la Constitución republicana. La noticia se puede consultar aquí: https://elpais.com/diario/1978/11/29/espana/281142001_850215.html. Sobre las vicisitudes del manifiesto y la Plataforma, se puede consultar MORENO SECO, Manifiestos feministas. Antología de textos del movimiento feminista español (1965-1985). Universidad de Alicante, 2005. p. 174; GAHETE MUÑOZ, Soraya: Por un feminismo radical y marxista. El Colectivo Feminista de Madrid en el contexto de la Transición española (1976-1980). Tesis doctoral, Universidad Complutense de Madrid, 2018, p. 311 y RODRÍGUEZ PALOP, "Esta Constitución no es nuestra ...", cit., pp. 138 y ss.

23 MORENO SECO, “Manifiestos feministas...”, cit., pp. 175-176.

24 Es por ello igualmente sorprendente afirmar, como hace Rodríguez Palop, que: “... el movimiento feminista organizado acabó militando en favor de la abstención en el referéndum constitucional"; RODRÍGUEZ PALOP, 2018, cit., p. 140. Véase, en contraste, MORENO SECO, Mónica: "Mujer y culturas políticas en el franquismo y el 
La segunda razón por la que es estrambótica la afirmación de la Plataforma de Organizaciones Feministas de Madrid de finales de noviembre de 1978 de acuerdo con la cual, para las mujeres la Constitución de 1978 era peor que la de sus abuelas de 1931, radica en el hecho de que la Constitución de la República española de 1931 sólo pudo ser votada por "dos abuelas", las dos únicas diputadas que se sentaban en el Congreso de los Diputados, elegidas cuando aún las mujeres no tenían en España derecho al sufragio activo ${ }^{25}$. La Constitución española de 1978, sin embargo, pudo ser refrendada por todas las mujeres españolas mayores de 18 años.

De lo anterior conviene extraer dos lecciones importantes: (1) la participación activa de las mujeres en el proceso constituyente no es condición necesaria ni suficiente para que los contenidos de una Constitución contribuyan a la causa del feminismo ${ }^{26}$ (del mismo modo que ocurre, por cierto, con la participación de miembros de los pueblos originarios de Chile que se pretende asegurar mediante la reserva de $\operatorname{cuota}^{27}$ ). No de otra forma cabe entender que la Constitución española de 1931 pueda ser mejor para las mujeres que la de 1978, o que, como más modernamente destacan Rubio-Marín e Irving refiriéndose a los procesos constituyentes en países de religión musulmana (Marruecos, Túnez, Egipto), la participación de las mujeres no garantiza una mejor protección de sus derechos ${ }^{28}$. ¿Sería más favorable para el conjunto de las mujeres chilenas que la Convención Constitucional tuviera una composición paritaria pero que las mujeres fueran todas similares a la muy controvertida conservadora Macarena Santelices?; y (2) una Constitución, como señalaban las autoras del manifiesto en el que se solicitaba el "sí" para la Constitución española de 1978, no debe reflejar las aspiraciones particulares de un grupo de la población, por muy

antifranquismo". En: Pasado y Memoria. Revista de Historia Contemporánea, Vol. 7, 2008. p. 185) y GAHETE MUÑOZ, cit., pp. 314 y ss.

25 Fue precisamente la Constitución finalmente aprobada el 9 de diciembre de 1931 la encargada de consagrarlo, con la oposición de una de las dos "abuelas", la diputada socialista Victoria Kent.

26 Para las referencias concretas en experiencias constitucionales recientes, véase RUBIOMARÍN y IRVING, cit., pp. 11-15.

27 Cuando estas páginas terminan de escribirse, el Senado chileno ha aprobado una primera norma que establece un número de 23 escaños a añadir a los 155 de la Convención Constitucional para los pueblos originarios, frente a la posibilidad de reservar una cuota dentro de aquéllos 155 que figuran en el acuerdo de 15 de noviembre de 2019. La votación definitiva sobre el mecanismo de representación se producirá el 10 de noviembre de 2020. Véase al respecto: https://www.senado.cl/despachan-reformasobre-escanos-reservados-para-pueblos-originarios-e/senado/2020-10-29/230654.html.

28 Ibid., pp. 16-17. 
identificable que pueda ser, sino que ha de tener vocación de perdurar como Magna Carta para todos los ciudadanos.

Establecer un candado de paridad futura, como señalaba antes, puede tener justificación como forma de solidificar una imagen social maestra: las mujeres que, como se suele repetir, son "la mitad de todo" 29 , han de ser paritariamente protagonistas en la "estructura básica de la sociedad", por emplear la conocida terminología de John Rawls. Ese candado, empero, puede operar como un indeseable escollo si, de nuevo, la opción que prospera es la del detalle de una regla más que el efecto de delegación que tienen los principios abiertos. Y es que una cosa es fijar una paridad estricta que sigue a la división demográfica estadísticamente habitual entre hombres y mujeres $(50 \%)$ y otra muy distinta es constitucionalizar una "presencia equilibrada" entre hombres y mujeres. Por razones suficientemente obvias de flexibilidad y operatividad parece mucho más recomendable consagrar constitucionalmente la segunda y no la primera fórmula.

Conviene recordar, además, que el establecimiento de cuotas que operan frente al criterio meritocrático o que alteran sustancialmente la libertad de elección de los individuos o las asociaciones, se ha visto siempre como una medida transitoria, que debe perdurar en tanto los obstáculos, sesgos e inercias que impiden la representación equilibrada se disipan y los resultados en términos de presencia de las mujeres se hacen aceptables mediante los mecanismos intervenidos ${ }^{30}$. Ese carácter "transitorio" es, nuevamente, contrario a la vocación de perdurabilidad que acompaña a las Constituciones.

La Constitución chilena, como cualquier otro texto constitucional, debería tener, a mi juicio, el afán de servir de instrumento para organizar el poder del Estado y garantizar los derechos y libertades básicos de una ciudadanía cuyos miembros son esencialmente "iguales aunque distintos". Es posible que la representación de la plurinacionalidad y la inclusión de las mujeres en la Convención Constitucional sirvan mejor a ese fin, entre otras cosas por la fuerza simbólica de esa composición plural. Consagrar, sin embargo, un constitucionalismo de fragmentación identitaria a partir de la

29 Sobre las consecuencias normativas varias que se sigue de esa "presunta" obviedad, véase PHILIPS, Ann: "Defending Equality of Outcome". En: Journal of Political Philosophy, Vol. 12, nº $1,2004$.

30 Coincido con Alfonso Ruiz Miguel cuando señala que las cuotas electorales no plantean un problema de "discriminación" atentatoria a la igualdad, sino que el valor sacrificado es el de la libertad. Véase RUIZ MIGUEL, Alfonso: "En defensa de las cuotas electorales para la igualdad de las mujeres". En: Aequalitas, N²0, 2007. pp. 63-65. 
consideración de que los individuos son esencialmente miembros adscritos a colectivos por rasgos que escapan a su voluntad ${ }^{31}$, puede suponer un indeseable retorno a las medievales sociedades estamentales.

\section{CONCLUSIONES: ELTRILEMADELCONSTITUCIONALISMO FEMINISTA.}

A lo largo de las anteriores páginas he tratado de dar respuesta a las cuestiones relativas a lo que puede significar una Constitución con perspectiva de género, o feminista o paritaria. Al paso de ese análisis he ido advirtiendo algunas aporías, y es ahora, al concluir el ensayo, que me atrevo a formular que el constitucionalismo feminista, con los componentes que he descrito, ha de enfrentarse a un trilema: si la Constitución es feminista porque consagra alguna de las demandas concretas del movimiento feminista que son socialmente disolventes, entonces esa Constitución traicionará algunos presupuestos básicos del constitucionalismo (la perdurabilidad y su carácter de amplio acuerdo social); si la Constitución es feminista porque reivindica la igualdad de ciudadanía entre hombres y mujeres, la dimensión feminista no aporta nada toda vez que esa es ya una conquista sobre la que no cabe "hoja en blanco"; si la Constitución es feminista porque promueve que se alcance la igualdad real con expresiones ideológicas que trascienden al feminismo, la Constitución no será necesariamente feminista.

Así pues, o inexistencia de Constitución, o futilidad o carácter innecesario del feminismo; he ahí el trilema.

31 No puedo dejar de mencionar, siquiera sea sumariamente, que la constitucionalización de la paridad, si es que no la paridad constituyente misma, es difícilmente compatible en sus propósitos con la auto-identificación de género que con tanta fuerza se está esgrimiendo como reivindicación nuclear del colectivo LGTBIQ+. Sobre ello me permito remitirme al capítulo 6 de mi libro Lo sexual es político (y jurídico). Pero esas mismas razones que aplican a la "auto-identificación" de género habrían de predicarse también de las posibilidades de "auto-identificación" como miembro de un pueblo originario que permite la actual legislación chilena sobre la materia: véase el Decreto 392 del Ministerio de Planificación y Cooperación de 24 de noviembre de 1993 (https:// www.bcn.cl/leychile/navegar?idNorma=12976). Agradezco a Ignacio Ackermann el haberme descubierto estas valiosas fuentes documentales y algunas otras claves para entender mejor el proceso constituyente chileno. 


\section{BIBLIOGRAFÍA.}

CORREA FLOREZ, María: Legítima defensa en situaciones sin confrontación: la muerte del tirano de casa. Colección Ciencias Penales, Grupo Editorial Ibáñez, Universidad de los Andes, Bogotá, 2017.

DE LORA, Pablo: Lo sexual es político (y jurídico). Alianza, Madrid, 2019.

FIELD BELENKY, Mary, MCVICKER CLINCHY, Blythe, RULE GOLDBERGER, Nancy, y MATTUCK TARULE, Jill: Women's Ways of Knowing: The Development of Self, Voice, and Mind, Basic Books, New York, 1986.

GAHETE MUÑOZ, Soraya: Por un feminismo radical y marxista. El Colectivo Feminista de Madrid en el contexto de la Transición española (1976-1980). Tesis doctoral, Universidad Complutense de Madrid, 2019.

GALLIE, W. B.: "Essentially Contested Concepts". En: Proceedings of the Aristotelian Society, Vol. 56, 1955. Págs. 167-198.

GARCÍAFIGUEROA, Alfonso: "Feminismo de Estado: fundamentalmente religioso y religiosamente fundamentalista". En: Eunomía. Revista en Cultura de la Legalidad, 17, 2019. Págs. 358-376.

GIMENO, Beatriz: "Cambiarlo todo". En: Ctxt, N¹58, 2018, febrero (https://ctxt.es/es/20180228/Firmas/18208/Feminismo-izquierdapuritanismo-neoliberalismo-Beatriz-Gimeno.htm).

GÓMEZ FERNÁNDEZ, Itziar: “¿Qué es eso de reformar la Constitución con perspectiva de género? Mitos caídos y mitos emergentes a partir del libro Una constituyente feminista”. En: Eunomía. Revista en la Cultura de la Legalidad, Vol. 16, 2019. Págs. 312-329.

LAGOS TSCHORNE, Catalina: “¿Por qué necesitamos una Constitución con perspectiva de género? ¿Y qué significa?”. En: Revista Emancipa, 6 de noviembre, 2020 (https://revistaemancipa.org/2020/11/06/por-quenecesitamos-una-constitucion-con-perpectiva-de-genero-y-que-significa/, última visita el 9 de noviembre de 2020).

MORENO SECO, Mónica: "Mujer y culturas políticas en el franquismo y el antifranquismo". En: Pasado y Memoria. Revista de Historia Contemporánea, Vol. 7, 2008. Págs. 165-185. 
MORENO SECO, Mónica: Manifiestos feministas. Antología de textos del movimiento feminista español (1965-1985). Universidad de Alicante, 2005.

OKIN, Susan Moller: Justice, Gender and the Family. Basic Books, 1989.

PHILIPS, Ann: "Defending Equality of Outcome". En: Journal of Political Philosophy, Vol. 12, nº1, 2004. Págs. 1-19.

PINKER, Steven: The Better Angels of Our Nature. Penguin, Nueva York, 2011.

RADCLIFFE-RICHARDS, Janet: "Only X\%: The Problem of Sexual Equality". En: Journal of Practical Ethics, Vol. 2, Issue 1, 2014. Págs. 44-67

RODRÍGUEZ PALOP, María Eugenia: "Esta Constitución no es nuestra. Propuestas para una Constitución feminista”. En: Escudero Alday, Rafael y Martín, Sebastián (eds.): Fraude o esperanza: 40 años de la Constitución. Akal, Madrid, 2018. Págs. 133-155.

RODRÍGUEZ PALOP, María Eugenia: "Argumentos contra la gestación por sustitución". En: García Manrique, Ricardo, (ed.): El cuerpo diseminado. Estatuto, uso y disposición de los biomateriales humanos. Aranzadi, Navarra, 2018a.

RUBIO-MARÍN, Ruth y IRVING, Helen (2019): "Women as ConstitutionMakers: The Promises and the Challenges of Participation". En: RubioMarín, Ruth y Irving, Helen (edits): Women as Constitution-Makers. Case Studies From the New Democratic Era. Cambridge University Press, Cambridge-Nueva York, 2019. Págs. 1-30.

RUIZ MIGUEL, Alfonso: "En defensa de las cuotas electorales para la igualdad de las mujeres”. En: Aequalitas, N²0, 2007. Págs. 60-68. 